\title{
Suboptimal response to $\mathrm{GnRH}$-agonist trigger during oocyte cryopreservation: a case series
}

Miguel Russo ${ }^{1,2^{*}}$, Kimberly Liu ${ }^{1,2}$ and Crystal Chan ${ }^{1,2,3}$

\begin{abstract}
Background: Random-start, controlled ovarian stimulation (COS) has advanced the field of fertility preservation, allowing patients to expedite fertility treatment and avoid further delays to their cancer therapy. This novel approach allows patients to initiate ovarian stimulation at any point, regardless of where they are in their menstrual cycle. Luteal-phase start (LPS) protocols describe treatment cycles where COS is initiated during the luteal-phase of the menstrual cycle. LPS protocols have not been studied or optimized to the same degree as conventional, earlyfollicular COS. Particularly, there is a paucity of evidence evaluating treatment outcomes using different trigger medications in LPS protocols. The present study aims to evaluate the efficacy of using a GnRH agonist (GnRH-a) trigger in patients undergoing oocyte cryopreservation in LPS protocols.

Methods: This descriptive case series describes two patients, recently diagnosed with cancer, who underwent oocyte cryopreservation using an LPS protocol and a GnRH-a trigger at a university-affiliated, academic center.

Results: The patients described in our case series both failed to adequately respond to a GnRH-a trigger, based on their serum levels of luteinizing hormone $(\mathrm{LH})$ and progesterone $12 \mathrm{~h}$ after their $\mathrm{GnRH}$-a trigger. They both required a single rescue dose of human chorionic gonadotropin (hCG).

Conclusions: These findings highlight the potential risk of a suboptimal response to a GnRH-a trigger in patients undergoing LPS, controlled ovarian stimulation for oocyte cryopreservation. This risk might be attributed to the downregulation of $\mathrm{GnRH}$ receptors by elevated serum progesterone levels during the luteal phase. Currently, there is insufficient evidence to recommend for or against the use of a GnRH-a trigger during LPS controlled ovarian stimulation. This case series offers a number of management strategies to mitigate this risk and emphasizes the need for further research in this area.
\end{abstract}

Keywords: Fertility preservation, Random-start controlled ovarian stimulation, Trigger medication, Suboptimal response, GnRH agonist, Luteal-phase start protocol

\footnotetext{
*Correspondence: miguel.russo@sinaihealth.ca

'Division of Gynaecologic Reproductive Endocrinology and Infertility,

Department of Obstetrics and Gynecology, Mount Sinai Hospital, University

of Toronto, Toronto, Canada

${ }^{2}$ Mount Sinai Fertility, 7th floor, 250 Dundas Street West, Toronto, Ontario

M5T 2Z5, Canada

Full list of author information is available at the end of the article
}

(C) The Author(s). 2020 Open Access This article is licensed under a Creative Commons Attribution 4.0 International License, which permits use, sharing, adaptation, distribution and reproduction in any medium or format, as long as you give appropriate credit to the original author(s) and the source, provide a link to the Creative Commons licence, and indicate if changes were made. The images or other third party material in this article are included in the article's Creative Commons licence, unless indicated otherwise in a credit line to the material. If material is not included in the article's Creative Commons licence and your intended use is not permitted by statutory regulation or exceeds the permitted use, you will need to obtain permission directly from the copyright holder. To view a copy of this licence, visit http://creativecommons.org/licenses/by/4.0/. The Creative Commons Public Domain Dedication waiver (http://creativecommons.org/publicdomain/zero/1.0/) applies to the data made available in this article, unless otherwise stated in a credit line to the data. 


\section{Introduction}

Conventionally, controlled ovarian stimulation (COS) for in-vitro fertilization (IVF) is started in the early follicular phase of the menstrual cycle. However, for cancer patients undergoing fertility preservation, this approach is being supplanted by random-start COS in order to help expedite urgent gonadotoxic chemotherapy. Random-start COS allows patients to start ovarian stimulation at any point during their menstrual cycle. Luteal phase start (LPS) is a specific type of random-start COS where patients begin stimulation in the luteal phase of their cycle, when progesterone levels are elevated.

The feasibility of random-start COS can be explained by the wave theory of follicular recruitment - a concept first described by Baerwald et al. [1] They demonstrated that two or more waves of follicle development can be recruited during a single physiologic, menstrual cycle. The efficacy of random-start COS was eventually validated by early studies demonstrating equivalent treatment outcomes when compared to conventional COS, for fertility preservation [2-4].

However, LPS protocols have not been studied or optimized to the same degree as conventional COS. There is a paucity of literature evaluating the efficacy of different trigger medications during LPS, particularly the use of a GnRH agonist (GnRH-a) to reduce the risk of ovarian hyperstimulation syndrome (OHSS). While reducing the risk of OHSS is critical to avoid delays to life-sparing cancer treatment, a suboptimal response to a GnRH-a trigger could lead to poor outcomes such as low oocyte maturity or empty-follicle syndrome (EFS) [5-11]. The present study explores the novel hypothesis that LPS may be a risk factor for a suboptimal response to a $\mathrm{GnRH}$-a trigger.

\section{Methods}

This is a descriptive, case series of two cycling, nulligravid patients undergoing oocyte cryopreservation (OC) at a tertiary, academic centre after a recent cancer diagnosis (see Table 1). Both patients underwent LPS COS using a $\mathrm{GnRH}$ antagonist protocol (Cetrotide $250 \mathrm{mcg}$ daily) with recombinant FSH (r-FSH). A GnRH agonist trigger (buserelin $0.5 \mathrm{mg}$ ) was administered once the patients met a pre-specified criteria ( $\geq 3$ follicles measuring $\geq 17 \mathrm{~mm}$ ). The choice of trigger was based primarily on the discretion of the physician. It was common practice at our centre to routinely use a GnRH-a trigger for the majority of oncofertility cases to mitigate the risk of OHSS. Routine bloodwork was performed $12 \mathrm{~h}$ posttrigger medication to confirm an adequate rise in luteinizing hormone (LH) and progesterone. Both patients demonstrated a suboptimal response (low serum luteinizing hormone (LH) and/or progesterone (P4) levels) and required a rescue dose of hCG (r-hCG 250 $\mathrm{mcg}$ ). Oocyte retrieval was performed $36 \mathrm{~h}$ after their rescue trigger, as per protocol. Written informed consent was obtained from each patient for research purposes and publication of their case study.

\section{Result(s)}

\section{Case 1: Hodgkin's lymphoma}

A 33-year-old nulligravid, single female was seen in consultation for consideration of fertility preservation after a recent diagnosis of Hodgkin's lymphoma. She was scheduled to start a chemotherapy regimen which included Adriamycin, Bleomycin, Vinblastine and Dacarbazine (ABVD). She had slightly irregular menstrual cycles lasting between 28 to 40 days.

Her antral follicle count (AFC) was 14 and antimullerian hormone (AMH) level was $5 \mathrm{pmol} / \mathrm{L}(0.7 \mathrm{ng} /$

Table 1 Demographics and Cycle Characteristics Summary

\begin{tabular}{lll}
\hline Patient Demographics and Cycle Characteristics & Case 1 & Case 2 \\
\hline Age (yrs) & 33 & 35 \\
Diagnosis & Hodgkin's Lymphoma & Triple-negative Breast cancer \\
Ovarian Reserve Testing & AMH $=5$ pmol/L; Random AFC $=14$ & No AMH; Random AFC $=11$ \\
P4 (nmol/L) & 6 & 16 \\
Total Days of Stimulation & 11 & 7 \\
Total Dose of FSH (IU) & $3375 I U$ & 2000 IU \\
E2 on Trigger Day (pmol/L) & 2690 & 1030 \\
No.of Follicles $>15$ mm on Trigger Day & 5 & 4 \\
LH (IU/L) & 53 & 3.7 \\
P4 (nmol/L) & 4 & 5 \\
Oocytes Retrieved & 9 & 7 \\
Mature Oocytes (MIls) & 8 & 7 \\
\hline
\end{tabular}

a Day 1 of gonadotropin stimulation

b $12 \mathrm{~h}$ post $\mathrm{GnRH}$-a trigger 
$\mathrm{mL})$. On Day 18 of her menstrual cycle, she had a leading follicle measuring $1.7 \mathrm{~cm}$ on her right ovary and her serum estradiol (E2) level was $350 \mathrm{pmol} / \mathrm{L}$, luteinizing hormone (LH) was $20 \mathrm{IU} / \mathrm{L}$ and her progesterone (P4) was $3 \mathrm{nmol} / \mathrm{L}$. These findings suggested that the patient was on the late-follicular phase. She was subsequently assessed 2 days later and her ultrasound showed a rightsided $2.1 \mathrm{~cm}$ corpus luteum. Furthermore, her E2 dropped to $221 \mathrm{pmol} / \mathrm{L}$, LH was $7.2 \mathrm{IU} / \mathrm{L}$ and progesterone was $6 \mathrm{nmol} / \mathrm{L}$, suggesting she was in the early luteal phase. She was started on $375 \mathrm{IU}$ of recombinant follicle stimulating hormone (rFSH) (Puregon, Merck, Kirkland, Canada). On Day 5 of gonadotropin stimulation, she was started on a GnRH antagonist (Ganirelix 250mcg sc, Merck, Kirkland, Canada) daily. After 11 days of gonadotropin stimulation, her E2 was $2690 \mathrm{pmol} / \mathrm{L}$, LH was 0.8 $\mathrm{IU} / \mathrm{L}$ and $\mathrm{P} 4$ was less than $1 \mathrm{nmol} / \mathrm{L}$. Transvaginal ultrasound (TVUS) showed 5 dominant follicles measuring $\geq 15 \mathrm{~mm}$ and she met pre-specified trigger criteria. She was triggered using $0.5 \mathrm{mg}$ of buserelin acetate (Suprefact, Sanofi-aventis, Laval, Canada). Bloodwork performed $12 \mathrm{~h}$ post $\mathrm{GnRH}$-a trigger revealed an LH of 53 $\mathrm{IU} / \mathrm{L}$ but a $\mathrm{P} 4$ of $4 \mathrm{nmol} / \mathrm{L}$. Based on these findings, she received a rescue dose of $250 \mu \mathrm{g}$ of recombinant choriogonadotropin alpha (r-hCG) (Ovidrel, EMD Serono, Mississauga, Canada) and had her oocyte retrieval $36 \mathrm{~h}$ after her initial GnRH-a trigger. At the time of her egg retrieval, there were 9 oocytes retrieved of which 8 were in Metaphase II and cryopreserved.

\section{Case 2: breast Cancer}

A 35-year-old nulligravid female was seen in consultation for consideration of fertility preservation after a recent diagnosis of triple negative, breast cancer (genetic testing pending). The patient was scheduled to start neoadjuvant chemotherapy using a dose dense Doxorubicin, Cyclophosphamide, and Paclitaxel (AC-PACL) as soon as possible. Her menstrual cycles were regular lasting approximately 28 days in length. Her past medical history was significant for hypothyroidism.

On the day of her consultation, she had an ultrasound which showed an AFC was 11 and a leading follicle on her left ovary measuring $1.6 \mathrm{~cm}$. Her bloodwork revealed an $\mathrm{E} 2$ was $856 \mathrm{pmol} / \mathrm{L}$, $\mathrm{LH}$ of $8.6 \mathrm{IU} / \mathrm{L}$, and a progesterone of $3 \mathrm{nmol} / \mathrm{L}$. She received a dose of $250 \mu \mathrm{g}$ of recombinant choriogonadotropin alpha (r-hCG) (Ovidrel, EMD Serono, Mississauga, Canada) to hasten ovulation and started on gonadotropin stimulation 4 days later. At the start of stimulation, her E2 was $624 \mathrm{pmol} / \mathrm{L}, \mathrm{LH}$ of $24 \mathrm{IU} / \mathrm{L}$, and $\mathrm{P} 4$ of $16 \mathrm{nmol} / \mathrm{L}$. Furthermore, her US showed a left-sided $1.4 \mathrm{~cm}$ corpus luteum. These findings suggested that the patient was in the luteal phase at the start of her ovarian stimulation. She was started on $250 \mathrm{IU}$ of rFSH (Puregon, Merck, Kirkland, Canada) and
$5 \mathrm{mg}$ of letrozole daily (Femara, Novartis, Dorval, Canada). On Day 5 of gonadotropin stimulation, she was started on a GnRH antagonist (Ganirelix 250mcg sc, Merck, Kirkland, Canada) daily.

After 7 days of gonadotropin stimulation, her E2 was $1030 \mathrm{pmol} / \mathrm{L}, \mathrm{LH}$ of $2.9 \mathrm{IU} / \mathrm{L}$, and $\mathrm{P} 4$ of $5 \mathrm{nmol} / \mathrm{L}$. Her ultrasound showed 4 follicles measuring $\geq 15 \mathrm{~mm}$ and she met pre-specified trigger criteria. She was triggered using $0.5 \mathrm{mg}$ of buserelin acetate (Suprefact, Sanofiaventis, Laval, Canada). Bloodwork performed $12 \mathrm{~h}$ post GnRH-a trigger revealed an LH of $3.7 \mathrm{IU} / \mathrm{L}$ but a P4 of $5 \mathrm{nmol} / \mathrm{L}$. Based on these findings, she received a rescue dose of $250 \mu \mathrm{g}$ of recombinant choriogonadotropin alpha (r-hCG) (Ovidrel, EMD Serono, Mississauga, Canada) and had her oocyte retrieval $36 \mathrm{~h}$ after her rescue trigger. During her egg retrieval, she had a total of 7 oocytes retrieved, all in Metaphase II. She had 3 oocytes cryopreserved and 4 oocytes fertilized with her partner's sperm using conventional IVF. She had 4 cleavage-stage embryos which were cultured to Day 5. On Day 5, she had 2 viable blastocysts which were cryopreserved.

\section{Discussion}

The present case series highlights the potential risk of a suboptimal response to a GnRH-a trigger during LPS. Failure to induce an adequate $\mathrm{LH}$ surge after a $\mathrm{GnRH}-\mathrm{a}$ trigger can result in poor follicular luteinisation, oocyte maturation and oocyte yield [5-11]. While controversy remains over the exact definition, a suboptimal response has been previously defined as a serum LH below $15 \mathrm{IU} /$ $\mathrm{L}$ or serum progesterone levels that fail to rise significantly 8 to $12 \mathrm{~h}$ post trigger [12]. This case series emphasizes the need to evaluate the use of a GnRH-a trigger in patients undergoing LPS, primarily in the context of fertility preservation, and explores the biological plausibility behind this hypothesis.

Currently, there is a paucity of data evaluating treatment outcomes using a GnRH-a trigger in patients undergoing LPS for fertility preservation. In the largest publication to date, Cakmak et al. [4] examined 144 fertility preservation cycles, using either conventional or random-start COS, and found no differences in treatment outcomes [4]. In this study, 63\% (22/35) of random-start cycles began during the luteal phase. Moreover, 28.2\% of random-start cycles used a GnRH-a trigger [4]; however, it is unclear whether any of these were luteal-phase starts. Therefore, it is difficult to estimate the efficacy of a GnRH-a trigger during LPS from this study.

Two case reports have also been published describing the use of a $\mathrm{GnRH}$-a trigger in patients undergoing fertility preservation using random-start COS. Nayak and Wakim [3] published a cases series of 4 patients undergoing random-start COS using a GnRH-a trigger, only 
one of which had a LPS protocol. The authors concluded that a GnRH-a trigger effectively triggers oocyte maturation despite having an overall oocyte maturity rate of $63 \%$ (61 MIIs/96 oocytes) [3]. Ozkaya et al. ${ }^{13}$ also published a case report describing a patient undergoing random-start ovarian stimulation for fertility preservation after a diagnosis of Hodgkin lymphoma. Ten hours after a GnRH-a trigger, her serum LH levels were 89 $\mathrm{mIU} / \mathrm{mL}$ and her serum progesterone levels rose to 9.4 $\mathrm{ng} / \mathrm{mL}(30 \mathrm{nmol} / \mathrm{L})$ suggesting an adequate response to her $\mathrm{GnRH}$-a trigger. However, her oocyte maturity rate was 52\% (17 MIIs/31 oocytes). The authors commented that, in their experience, they had observed a suboptimal LH surge post GnRH-a trigger in $1.3 \%(1 / 75)$ of cycles with random-start COS [13]. However, it is unclear what proportion of these cycles had LPS as patient demographics and cycle characteristics were not published.

The use of a GnRH-a trigger, as opposed to hCG, has become a common strategy to reduce the risk of OHSS in high risk patients. Compared to hCG, GnRH-a has a significantly shorter half-life, limiting the release of vasoactive peptides from granulosa cells, which are primarily responsible for the development of OHSS [14]. A $\mathrm{GnRH}-\mathrm{a}$ trigger functions by stimulating an endogenous surge of LH that leads to luteinisation of ovarian follicles and activates oocyte maturation into Metaphase II. Animal studies have demonstrated that the ability of a species to respond to $\mathrm{GnRH}$ is based primarily on the density of GnRH receptors (GnRH-r) expressed within the gonadotropes in the pituitary gland [15], which is highest just prior to ovulation [16]. This is likely a necessary pre-requisite for the pituitary to generate an $\mathrm{LH}$ surge and trigger the process of ovulation. Moreover, GnRH-r expression in the pituitary gland is regulated in part - by estrogen and progesterone. Estradiol has been shown to increase the expression and responsiveness of GnRH-r [17] while progesterone has been shown to have the opposite effect, with the lowest concentration of GnRH-r found during the luteal phase [18]. Moreover, the downregulation of GnRH-r mediated by progesterone appears to be dose- and timedependent [19].

During LPS, the corpus luteum has already developed and production of progesterone has begun. As the elevated progesterone levels begin to downregulate $\mathrm{GnRH}$ $r$, the rising estradiol levels from COS will begin to exert an opposite effect. Eventually, the inhibitory effect of progesterone is withdrawn by the initiation of a $\mathrm{GnRH}$ antagonist and resulting luteolysis. Serum progesterone levels begin to decrease as estradiol levels continue to rise, promoting upregulation of GnRH-r (see Fig. 1). However, it is plausible that - in some cases - there may be insufficient time for GnRH-r to reach the "crucial density" required to produce an optimal response to a GnRH-a trigger. Presently, this risk may be difficult to quantify given our limited knowledge about the expression of GnRH-r within humans. Likewise, it would be premature to conclude that the mere absence of "detectable" progesterone levels, as in these cases, at the time of trigger would eliminate this risk. It is possible that there might be a physiological lag time between when serum progesterone levels become undetectable and the upregulation of GnRH-r begins.

While there is limited evidence evaluating the use of a GnRH-a trigger during LPS, there are other clinical scenarios that support the risk of suboptimal response to a $\mathrm{GnRH}$-a trigger in the presence of elevated progesterone levels. Several studies have been published to evaluate the efficacy of progesterone for pituitary suppression during IVF treatment [20, 21]. In a study by Kuang et al. [20], authors prospectively compared IVF outcomes between patients using medroxyprogesterone acetate

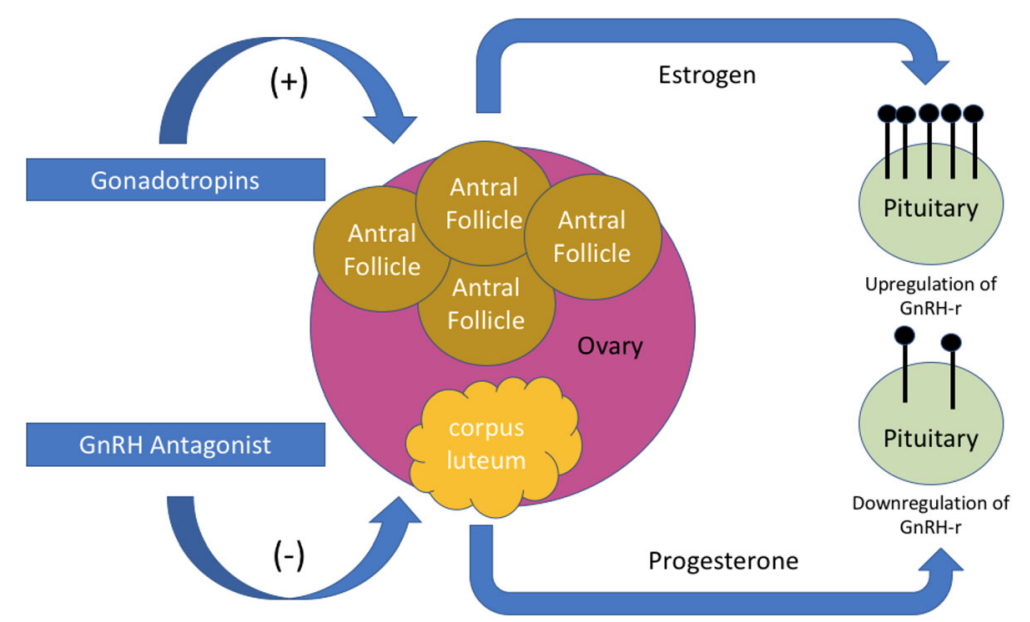

Fig. 1 GnRH-r Regulation during Luteal-Phase COS 
(MPA) for pituitary downregulation, compared to a standard, microdose-flare protocol. For patients in the MPA group, a GnRH-a trigger was used initially; however, during the early stages of their study, the authors observed a suboptimal LH response (less than $20 \mathrm{IU} / \mathrm{L}$ ) $10 \mathrm{~h}$ post-trigger in $5.7 \%$ of treatment cycles (3/53) [20]. These patients were subsequently found to have lower rates of oocyte maturity (0, 25 and 90\%). A retrospective study was subsequently published by the same group with the intent to identify risk factors for a suboptimal response to a $\mathrm{GnRH}$-a trigger (defined as an $\mathrm{LH} \leq 15$ $\mathrm{mIU} / \mathrm{mL}$ ) [21]. The authors examined 8960 IVF cycles with MPA or Utrogestan for pituitary downregulation, using either a $\mathrm{GnRH}$-a trigger alone or in combination with hCG (1000, 2000, or $5000 \mathrm{IU})$. The rate of suboptimal response to GnRH-a trigger was $2.71 \%(243 / 8970)$ [21]. Patients with a suboptimal response had lower oocyte retrieval rates (48\% vs $68 \%$ ) and fewer mature oocytes (4 vs. 8). Compared to GnRH-a alone, the addition of hCG - irrespective of dose - was associated with an improvement in the oocyte retrieval rate but not the oocyte maturity rate [21].

In this case series, other potential causes for a suboptimal response to a GnRH-a trigger could be considered. For example, it is plausible that chronic stress due to a cancer diagnosis can lead to a state of hypogonadotropic hypogonadism which could explain a suboptimal response to GnRH-a trigger. Moreover, the suboptimal response observed between our patients was slightly different. The first patient had a suboptimal progesterone response but an adequate LH response; whereas, the second patient had suboptimal responses in both $\mathrm{LH}$ and progesterone levels. A possible explanation for this difference is that the second patient had significantly higher serum progesterone levels at the start of her ovarian stimulation. These findings could be attributed to the use of r-hCG to hasten ovulation prior to gonadotropin stimulation. Administration of hCG could have led to a prolonged and sustained production of progesterone from the corpus luteum, resulting in a more substantial downregulation of GnRH-r.

There are several options available to help mitigate the risk of a suboptimal response to a $\mathrm{GnRH}$-a trigger, including: using an hCG trigger only for patients at low risk of OHSS; considering a "rescue trigger" using hCG if post-trigger bloodwork reveals a suboptimal response; or using a combination of a GnRH-a trigger with a low dose of hCG, commonly referred to as a "dual trigger". While the efficacy of a dual trigger has been predominantly studied in patients undergoing IVF treatment with conventional COS, preliminary studies have been reassuring [22, 23].

The decision on whether to use a dual trigger routinely or to give a rescue trigger only in the event of a suboptimal response is at the discretion of the physician, and depends on the patient's individual risk of OHSS. Our report indicates that administering a rescue hCG trigger in the context of suboptimal hormonal response to GnRH-a trigger in LPS cycles can lead to an excellent yield of mature oocytes.

Timing of oocyte retrieval after suboptimal response to a GnRH-a trigger is also an important consideration. Our first patient had her egg retrieval $36 \mathrm{~h}$ after her initial trigger due to the adequate $\mathrm{LH}$ rise despite a suboptimal progesterone response. In contrast, our second patient had her egg retrieval $60 \mathrm{~h}$ after her initial trigger because both $\mathrm{LH}$ and progesterone levels failed to respond appropriately. Despite these differences in timing, both patients had successful egg retrievals. This supports the practice of delaying oocyte retrieval only if there is a suboptimal LH surge after GnRH-a trigger [12].

\section{Conclusion}

Random-start COS has advanced the field of fertility preservation by allowing patients to start ovarian stimulation immediately, minimizing delays to their cancer treatment. However, further research is necessary to optimize treatment outcomes, such as evaluating the use of different trigger medications, particularly in the context of LPS. The present case series elucidates the plausibility of a suboptimal response to a GnRH-a trigger during LPS and provides management strategies to mitigate this risk. Currently, there is insufficient evidence to recommend for or against the use of a GnRH-a trigger alone in patients undergoing fertility preservation with LPS. Therefore, the theoretical risk of suboptimal oocyte maturation or EFS with GnRH-a trigger alone has to be balanced with the potential increased risk of OHSS by routinely using a dual trigger. In the meantime, the authors of this study recommend careful consideration when using a GnRH-a trigger alone in patients undergoing LPS, and ensuring that an adequate response is documented prior to oocyte retrieval.

\section{Abbreviations \\ ABVD: Adriamycin, bleomycin, vinblastine and dacarbazine; AC- PACL: Doxorubicin, cyclophosphamide, and paclitaxel; AFC: Antral follicle count; AMH: Anti-mullerian hormone; COS: Controlled ovarian stimulation; EFS: Empty-follicle syndrome; GnRH-a: GnRH agonist; GnRH-r: GnRH receptors; hCG: Human chorionic gonadotropin; IVF: In-vitro fertilization; LH: Luteinizing hormone; LPS: Luteal-phase start; MII: Metaphase II; MPA: Medroxyprogesterone acetate; OC: Oocyte cryopreservation; OHSS: Ovarian hyperstimulation syndrome; P4: Progesterone; $r$ - FSH: Recombinant FSH; r-hCG: Recombinant hCG}

\section{Acknowledgements}

Not applicable.

Authors' contributions

All authors contributed substantially to the conception, design, literature review, drafting and revision of this manuscript and gave final approval for the version being submitted. 


\section{Funding}

Not applicable.

\section{Availability of data and materials}

The datasets used and/or analysed during the current study are available from the corresponding author on reasonable request.

\section{Ethics approval and consent to participate}

Written consent was provided by all patients included in this descriptive study.

\section{Consent for publication}

Written consent was provided by both patients included in this descriptive study.

\section{Competing interests}

The authors declare that they have no competing interests.

\section{Author details}

'Division of Gynaecologic Reproductive Endocrinology and Infertility, Department of Obstetrics and Gynecology, Mount Sinai Hospital, University of Toronto, Toronto, Canada. ${ }^{2}$ Mount Sinai Fertility, 7th floor, 250 Dundas Street West, Toronto, Ontario M5T 2Z5, Canada. ${ }^{3}$ Samuel Lunenfeld Research Institute, Toronto, Canada.

Received: 16 October 2019 Accepted: 20 May 2020

\section{Published online: 05 June 2020}

\section{References}

1. Baerwald, A. R., Adams, G. P., \& Pierson, R. A. (2004). Characterization of ovarian follicular wave dynamics in Women1. Biol Reprod, 69(3), 1023-1031. http://doi.org/https://doi.org/10.1095/biolreprod.103.017772

2. Sönmezer, M., Türküolu, I., Cokun, U., \& Oktay, K. (2011). Random-start controlled ovarian hyperstimulation for emergency fertility preservation in letrozole cycles. Fertil Steril, 95(6), 2125.e9-2125.e11. http://doi.org/https:// doi.org/10.1016/j.fertnstert.2011.01.030.

3. Nayak, S. R., \& Wakim, A. N. (2011). Random-start gonadotropin-releasing hormone $(\mathrm{GnRH})$ antagonist-treated cycles with $\mathrm{GnRH}$ agonist trigger for fertility preservation. Fertil Steril, 96(1), e51-e54. http://doi.org/https://doi. org/10.1016/j.fertnstert.2011.04.079

4. Cakmak, H., Katz, A., Cedars, M. I., \& Rosen, M. P. (2013). Effective method for emergency fertility preservation: random-start controlled ovarian stimulation. Fertil Steril, 100(6), 1673-1680. http://doi.org/https://doi.org/10. 1016/j.fertnstert.2013.07.1992.

5. Engmann, L., DiLuigi, A., Schmidt, D., Nulsen, J., Maier, D., \& Benadiva, C. (2008). The use of gonadotropin-releasing hormone $(\mathrm{GnRH})$ agonist to induce oocyte maturation after cotreatment with $\mathrm{GnRH}$ antagonist in highrisk patients undergoing in vitro fertilization prevents the risk of ovarian hyperstimulation syndrome: a prospective rando. Fertil Steril, 89(1), 84-91. http://doi.org/https://doi.org/10.1016/j.fertnstert.2007.02.002.

6. Melo M, Busso CE, Bellver J, Alama P, Garrido N, Meseguer M, et al. GnRH agonist versus recombinant HCG in an oocyte donation programme: a randomized, prospective, controlled, assessor-blind study. Reprod BioMed Online. 2009;19:486-92.

7. Sismanoglu A, Tekin HI, Erden HF, Ciray NH, Ulug U, Bahceci M. Ovulation triggering with GnRH agonist vs hCG in the same egg donor population undergoing donor oocyte cycles with $\mathrm{GnRH}$ antagonist: a prospective randomized cross-over trial. J Assist Reprod Genet. 2009;26:251-6.

8. Youssef MA, Van der Veen F, Al-Inany HG, et al. Gonadotropin-releasing hormone agonist versus HCG for oocyte triggering in antagonist-assisted reproductive technology. Cochrane Database Syst Rev. 2014;(10):CD008046. Published 2014 Oct 31. https://doi.org/10.1002/14651858.CD008046.pub4.

9. Humaidan, P., Kol, S., \& Papanikolaou, E. G. (2011). GnRH agonist for triggering of final oocyte maturation: time for a change of practice? Hum Reprod Update, 17(4), 510-524. http://doi.org/https://doi.org/10.1093/ humupd/dmr008.

10. Kummer, N. E., Griffin, D. W., Feinn, R. S., Nulsen, J. C., Engmann, L. L. \& Benadiva, C. A. (2012). Predicting successful induction of oocyte maturation after gonadotropin-releasing hormone agonist (GnRHa) trigger. Hum Reprod, 28(1), 152-159. http://doi.org/https://doi.org/10.1093/humrep/ des361.
11. Meyer, L., Murphy, L. A., Gumer, A., Reichman, D. E., Rosenwaks, Z., \& Cholst, N. N. (2015). Risk factors for a suboptimal response to gonadotropin-releasing hormone agonist trigger during in vitro fertilization cycles. Fertil Steril, 104(3), 637-642. http://doi.org/https://doi.org/10.1016/j.fertnstert.2015.06. 011.

12. Haas, J., \& Bentov, J. (2015). iMedPub Journals The Management of SubThreshold Serum LH Post GnRH Agonist Trigger Using serum progesterone as a surrogate Adding an HCG injection the day before the OPU Identifying patients with a propensity The use of $\mathrm{GnRH}$ agonist trigger significantly, 13. http://doi.org/https://doi.org/10.21767/2476-2008.10001.

13. Ozkaya, E., Roman, G. S., \& Oktay, K. (2012). Luteal phase GnRHa trigger in random start fertility preservation cycles. J Assist Reprod Genet, 29(6), 503505. http://doi.org/https://doi.org/10.1007/s10815-012-9752-8.

14. Corbett, S., On, O., Claman, P., Cheung, A., Bc, V., Sierra, S., ... Wong, B. (2014). SOGC CLINICAL PRACTICE GUIDELINE the prevention of ovarian Hyperstimulation syndrome. J Obstet Gynaecol Can, 36(11), 1024-1033. http://doi.org/https://doi.org/10.1016/S1701-2163(15)30417-5.

15. Rispoli, L. A., \& Nett, T. M. (2005). Pituitary gonadotropin-releasing hormone $(\mathrm{GnRH})$ receptor: structure, distribution and regulation of expression. Anim Reprod Sci, 88(1-2 SPEC. ISS), 57-74. http://doi.org/https://doi.org/10.1016/j. anireprosci.2005.05.004.

16. Nett TM, Turzillo AM, Baratta M, Rispoli LA. Pituitary effects of steroid hormones on secretion of follicle-stimulating hormone and luteinizing hormone. Dom Anim Endocrinol. 2002:23:33-42.

17. Schoenemann HM, Humphrey WD, Crowder ME, Nett TM, Reeves JJ. Pituitary luteinizinghormone-releasing hormone receptors in ovariectomized cows after challenge with ovarian steroids. Biol Reprod. 1985;32:574-83.

18. Turzillo AM, Clapper JA, Moss GE, Nett TM. Regulation of ovine GnRH receptor gene expression by progesterone and oestradiol. J Reprod Fertil. 1998a;113:251-6.

19. An, B. S., Choi, J. H., Choi, K. C., \& Leung, P. C. K. (2005). Differential role of progesterone receptor isoforms in the transcriptional regulation of human gonadotropin-releasing hormone I (GnRH I) receptor, $\mathrm{GnRH}$ I, and $\mathrm{GnRH}$ II. J Clin Endocrinol Metab, 90(2), 1106-1113. http://doi.org/https://doi.org/10. 1210/jc.2004-0318.

20. Kuang, Y., Chen, Q., Fu, Y., Wang, Y., Hong, Q., Lyu, Q., ... Shoham, Z. (2015). Medroxyprogesterone acetate is an effective oral alternative for preventing premature luteinizing hormone surges in women undergoing controlled ovarian hyperstimulation for in vitro fertilization. Fertil Steril, 104(1), 62-70. e3. http://doi.org/https://doi.org/10.1016/j.fertnstert.2015.03.022.

21. Lu, X., Hong, Q., Sun, L. H., Chen, Q., Fu, Y., Ai, A., ... Kuang, Y. (2016). Dual trigger for final oocyte maturation improves the oocyte retrieval rate of suboptimal responders to gonadotropin-releasing hormone agonist. Fertil Steril, 106(6), 1356-1362. http://doi.org/https://doi.org/10.1016/j.fertnstert. 2016.07.1068.

22. Griffin, D., Benadiva, C., Kummer, N., Budinetz, T., Nulsen, J., \& Engmann, L. (2012). Dual trigger of oocyte maturation with gonadotropin-releasing hormone agonist and low-dose human chorionic gonadotropin to optimize live birth rates in high responders. Fertil Steril, 97(6), 1316-1320. http://doi. org/https://doi.org/10.1016/j.fertnstert.2012.03.015.

23. O'Neill, K. E., Senapati, S., Maina, I., Gracia, C., \& Dokras, A. (2016). GnRH agonist with low-dose hCG (dual trigger) is associated with higher risk of severe ovarian hyperstimulation syndrome compared to GnRH agonist alone. J Assist Reprod Genet, 33(9), 1175-1184. http://doi.org/https://doi. org/10.1007/s10815-016-0755-8.

\section{Publisher's Note}

Springer Nature remains neutral with regard to jurisdictional claims in published maps and institutional affiliations. 